\title{
Pionic Deuterium
}

Th. Strauch ${ }^{1}$, F. D. Amaro 2 , D. F. Anagnostopoulos 3 , P. Bühler ${ }^{4}$, D. S. Covita ${ }^{2 a}$, H. Gorke ${ }^{5}$, D. Gotta ${ }^{1 b}$, A. Gruber $^{4}$, A. Hirtl $^{4 c}$, P. Indelicato ${ }^{6}$, E.-O. Le Bigot ${ }^{6}$, M. Nekipelov ${ }^{1}$, J. M. F. dos Santos ${ }^{2}$, Ph. Schmid ${ }^{4}$, S. Schlesser ${ }^{6}$, L. M. Simons ${ }^{7}$, M. Trassinelli ${ }^{6 \mathrm{~d}}$ J. F. C. A. Veloso ${ }^{8}$, and J. Zmeskal ${ }^{4}$

1 Institut für Kernphysik, Forschungszentrum Jülich, D-52425 Jülich, Germany

2 Dept. of Physics, Coimbra University, P-3000 Coimbra, Portugal

3 Dept. of Materials Science and Engineering, University of Ioannina, GR-45110 Ioannina, Greece

4 Stefan Meyer Institut for Subatomic Physics, Austrian Academy of Sciences, A-1090 Vienna, Austria

5 Zentralabteilung für Elektronik, Forschungszentrum Jülich GmbH, D-52425 Jülich, Germany

6 Laboratoire Kastler Brossel, UPMC-Paris 6, ENS, CNRS; Case 74, 4 place Jussieu, F-75005 Paris, France

7 Laboratory for Particle Physics, Paul Scherrer Institut, CH-5232 Villigen, Switzerland

8 Dept. of Physics, Aveiro University, P-3810 Aveiro, Portugal

\begin{abstract}
The strong interaction shift $\epsilon_{1 s}^{\pi D}$ and broadening $\Gamma_{1 s}^{\pi D}$ in pionic deuterium have been remeasured with high statistics by means of the $\pi \mathrm{D}(3 p-1 s) \mathrm{X}$-ray transition using the cyclotron trap and a high-resolution crystal spectrometer. Preliminary results are $\epsilon_{1 s}^{\pi D}=(-2325 \pm 31) \mathrm{meV}$ (repulsive) and $\Gamma_{1 s}^{\pi D}\left(1171_{-49}^{+23}\right) \mathrm{meV}$ which yields for the $\pi \mathrm{D}$ scattering length $a_{\pi D}=\left[-(24.8 \pm 0.3)+i\left(6.3_{-0.3}^{+0.1}\right)\right] \cdot 10^{-3} m_{\pi}^{-1}$. From the imaginary part the threshold parameter for pion production is determined to be $\alpha=\left(252_{-11}^{+5}\right) \mu \mathrm{b}$.
\end{abstract}

\section{Introduction}

The deuterium experiment is embedded in a series of measurements [1-3] aiming at a precision determination of the pion-nucleon scattering length comprising

- pionic hydrogen $(\pi \mathrm{H})$,

- pionic deuterium $(\pi \mathrm{D})$,

- muonic hydrogen $(\mu \mathrm{H})$, and

- the characterisation of the Bragg crystal spectrometer using narrow $\mathrm{X}$-ray lines from few-electron atoms produced by means of an electron-cyclotron resonance trap (ECRIT).

In this contribution, first results of the $\pi \mathrm{D}$ measurement are presented.

\section{Strong-interaction effects}

The complex pion-nucleus scattering length $a_{\pi A}$ of a pionic atom formed with a nucleus $A(Z, N)$ is related in leading

\footnotetext{
${ }^{a}$ present address: Dept. of Physics, Aveiro University, P-3810 Aveiro, Portugal

b corresponding author: d.gotta@fz-juelich.de

${ }^{c}$ present address: Universitätsklinik für Nuklearmedizin, Medizinische Universität Wien, 1090 Vienna, Austria

d present address: Inst. des NanoSciences de Paris, CNRS UMR7588 and UMPC-Paris 6, F-75015 Paris, France
}

order to the $1 \mathrm{~s}$-state shift $\epsilon_{1 s}$ and width $\Gamma_{1 s}$ by the Deser formula [4]

$$
\epsilon_{1 s}-i \frac{\Gamma_{1 s}}{2}=-\frac{2 \alpha^{3} \mu^{2} c^{4}}{\hbar c} a_{\pi A}+\ldots
$$

Ellipsis stand for corrections to the Deser formula because $a_{\pi D}$ is determined from a Coulomb bound state [5].

In $\pi \mathrm{H}$, the ground-state shift and broadening may be related to the $\pi N$ isoscalar and isovector scattering lengths $a^{+}$and $a^{-}$by

$$
\begin{gathered}
\epsilon_{1 s}^{\pi H} \propto a_{\pi^{-} p \rightarrow \pi^{-} p}=a^{+}+a^{-}+\ldots \\
\Gamma_{1 s}^{\pi H} \propto\left(a_{\pi^{-} p \rightarrow \pi^{0} n}\right)^{2}=2\left(a^{-}\right)^{2}+\ldots .
\end{gathered}
$$

In this case, $\Gamma \propto a^{2}$ because the hadronic broadening is due to a scattering process - the charge-exchange reaction $\pi^{-} p \rightarrow \pi^{0} n[6]$. The corrections here have been calculated in various approaches and amount up to a few per cent of the leading contribution and include to some extent already electromagnetic and strong isospin-breaking terms [7-12].

In the case of $\pi \mathrm{D}$, the real part $\mathfrak{R} a_{\pi D}$ of the scattering length, being proportional to the hadronic shift $\epsilon_{1 s}^{\pi D}$, maybe written by regarding the deuteron as a free proton and neutron as leading order and then applying additional corrections. One may write

$$
\begin{array}{rlrl}
\mathfrak{R} a_{\pi D} & =a_{\pi^{-} p \rightarrow \pi^{-} p}+a_{\pi^{-} n \rightarrow \pi^{-} n} & +\ldots \\
& = & 2 a^{+} & +\ldots
\end{array}
$$

This is an Open Access article distributed under the terms of the Creative Commons Attribution-Noncommercial License 3.0, which permits unrestricted use, distribution, and reproduction in any noncommercial medium, provided the original work is properly cited. 
where ellipsis stand for the higher order terms from multiple scattering (depending also on $a^{+}$and $a^{-}$), absorptive and possibly electromagnetic and strong isospin-breaking corrections. The second order correction is dominated by $\left(a^{-}\right)^{2}$ being comparable in magnitude to the leading order term $[13,14]$ because of the smallness of $a^{+}$as required by chiral symmetry $[15,16]$. The nuclear structure is taken into account by folding with the deuteron wave function.

The pionic deuterium shift provides a constraint on the $\pi N$ scattering lengths $a^{+}$and $a^{-}$. Its quality decisively depends on the precision of the data triple $\left(\epsilon_{1 s}^{\pi D}, \epsilon_{1 s}^{\pi H}, \Gamma_{1 s}^{\pi H}\right)$, for which a first set of precision data has been obtained in the last decade [17-20]. Vice versa, starting from the elementary pion-nucleon processes and involving properly nuclear structure, multiple scattering, and absorptive phenomena, the hadronic s-level shift of the $\pi \mathrm{D}$ system must be calculable unambiguously [21-31].

The forthcoming results for $\pi \mathrm{H}$ from this measurement series will improve the constraint [12], in particular by a better determination of the hadronic broadening in $\pi \mathrm{H}$ [3]. Finally, the accuracy for $a^{+}$will be determined mainly by the poorly known low-energy constant $f_{1}$ [12].

Secondly, $\pi \mathrm{D}$ gives access to pion absorption and production $N N \leftrightarrow N N \pi$ at threshold [32]. The hadronic broadening $\Gamma_{1 s}^{\pi D}$ mainly is due to true absorption $\left(\pi^{-} d \rightarrow n n\right)$ being the inverse and charge symmetric reaction of pion production $\left(p p \rightarrow \pi^{+} d\right)$ - in contrast to $\pi \mathrm{H}$ where the width is exclusively due to charge-exchange and radiative capture.

The relative strength of true absorption to radiative capture $\left(\pi^{-} d \rightarrow n n \gamma\right)$ was measured to $S=\frac{n n}{n n \gamma}=2.83 \pm$ 0.04 [33], and the branching ratio of internal pair conversion $\left(\pi^{-} d \rightarrow n n e^{+} e^{-}\right)$and charge exchange $\left(\pi^{-} d \rightarrow n n \pi^{0}\right)$ was found to be $0.7 \%$ [34] and $1.45 \pm 0.19 \cdot 10^{-4}$ [35], respectively. Hence, the relative strength of the true absorption channel to all other processes is obtained to $S^{\prime}=$ $n n /\left(n n \gamma+n n e^{+} e^{-}+n n \pi^{0}\right)=2.76 \pm 0.04$, i. e., about $2 / 3$ of the hadronic width and with that of the imaginary part $\mathfrak{J} a_{\pi D}$ is related to the process $\pi^{-} d \rightarrow n n$, which can be linked with pion production.

Pion production at low energies is usually parametrised by [36]

$$
\sigma_{p p \rightarrow \pi^{+} d}=\alpha C_{0}^{2} \eta+\beta C_{1}^{2} \eta^{3}+\ldots
$$

where $\eta=p_{\pi}^{*} / M_{\pi}$ is the reduced momentum of the pion in the $\pi d$ rest frame. For $\eta \rightarrow 0$ higher partial waves $(\beta, \ldots)$ vanish and only the threshold parameter $\alpha$ contributes representing pure s-wave production. The correction factors $C_{i}$ take into account the Coulomb interaction. $C_{0}^{2}$ is of the order of $30 \%[37,38]$ and an important source of uncertainty in the determination of $\alpha$ from cross-section data.

Another approach to determine $\alpha$ is to exploit the $\pi \mathrm{D}$ ground state broadening where uncertainties stemming from Coulomb correction factors and normalisation of cross sections are avoided. To derive the relation between $\alpha$ and $\mathfrak{J} a_{\pi D}$ as obtained from pionic deuterium, purely hadronic (non-experimental) cross sections $\tilde{\sigma}$ are introduced to circumvent the problem of diverging Coulomb cross section at threshold. Detailed balance relates pion production and absorption by

$$
\tilde{\sigma}_{\pi^{+} d \rightarrow p p}=\frac{2}{3} \cdot\left(\frac{p_{p}^{*}}{p_{\pi}^{*}}\right)^{2} \cdot \tilde{\sigma}_{p p \rightarrow \pi^{+} d}
$$

with $p_{p}^{*}$ and $p_{\pi}^{*}$ being final state center-of-mass (CMS) momenta [32]. Neglecting Coulomb and isospin breaking corrections, which are assumed to be at most a few per cent [39], charge symmetry requires equal strength for the transitions $\pi^{-} d \rightarrow n n$ and $\pi^{+} d \rightarrow p p$.

Combining optical theorem, charge invariance, detailed balance and inserting the parametrisation of the $p p \rightarrow \pi^{+} d$ cross section (4), the imaginary part of the $\pi^{-} d \rightarrow n n$ scattering length is related to the threshold parameter $\alpha$ by

$$
\begin{aligned}
\mathfrak{J} a_{\pi D} & =\left(1+\frac{1}{S^{\prime}}\right) \cdot \mathfrak{J} a_{\pi^{-} d \rightarrow n n} \\
& =\left(1+\frac{1}{S^{\prime}}\right) \cdot \frac{p_{\pi}^{*}}{4 \pi} \cdot \tilde{\sigma}_{\pi^{-} d \rightarrow n n} \\
& =\left(1+\frac{1}{S^{\prime}}\right) \cdot \frac{1}{6 \pi} \cdot \frac{\left(p_{p}^{*}\right)^{2}}{m_{\pi}} \cdot \alpha .
\end{aligned}
$$

The factor $\left(1+1 / S^{\prime}\right)$ corrects for the non true absorption channels.

For comparison of pionic-atom and pion-production data, the channels $p p \rightarrow \pi^{+} d$ and $n p \rightarrow \pi^{0} d$ can be used because in the limit of charge independence $2 \cdot \sigma_{n p \rightarrow \pi^{0} d}=$ $\sigma_{p p \rightarrow \pi^{+} d}$. Restricting to s waves, in both reactions the same transition of the nucleon pair ${ }^{3} S_{1}(I=0) \rightarrow{ }^{3} P_{1}(I=1)$ occurs which corresponds also to pion absorption at rest on the deuteron's isospin 0 nucleon-nucleon pair.

\section{Atomic cascade}

After pion capture in hydrogen isotopes, a quantum cascade starts from main quantum numbers at about $n \approx 16$. The upper and medium part of the de-excitation cascade is dominated by collisional processes (Stark mixing, external Auger effect, Coulomb de-excitation). In the lower part Xradiation becomes more and more important (Fig. 1) [4042]. Besides radiative de-excitation by X-ray emission all cascade processes depend on the environment, e.g., the density of the target gas.

Stark mixing essentially determines the X-ray yields in exotic hydrogen. Because these systems are electrically neutral and small on the atomic scale, they penetrate surrounding atoms and, thus, experience a strong Coulomb field. Non-vanishing matrix elements $\left\langle n l m|\mathbf{E}| n l^{\prime} m^{\prime}\right\rangle$ in the presence of the electric field mix atomic states of the same principle quantum number $n$ according to the selection rules $\Delta l= \pm 1$ and $\Delta m=0$ [43]. An induced s state, from where pions disapper by nuclear reactions, leads to a depletion of the cascade. The Stark mixing rate is proportional to the number of collisions during the exotic atom's life time and explains the strong decrease of the X-ray yields with increasing target density [40].

In the case of Coulomb de-excitation, the energy release of the de-excitation step is converted into kinetic energy of the collision partners [44]. For lower-lying transitions a significant energy gain occurs which leads to 
a Doppler broadening of subsequent X-ray transitions. Doppler broadening was directly observed first in the time-of-flight spectra of monoenergetic neutrons from the charge exchange reaction at rest $\pi^{-} p \rightarrow \pi^{0} n[45,46]$.

A dedicated measurement of the $\mu \mathrm{H}(3 p-1 s)$ transition has been performed within this series of experiments [47]. In $\mu \mathrm{H}$, where no hadronic broadening occurs, only the Doppler broadening exclusively from acceleration effects during the atomic cascade contributes and was directly observed as a significantly increased line width. The data were used - among others - to confirm that the methods used to quantify the corrections to the line width in pionic hydrogen and deuterium are sufficiently well defined [3]. Details may be found elsewhere [48].

The acceleration due to Coulomb de-excitation is counteracted by elastic and inelastic scattering. This leads to a rather complex velocity distribution with tails below the peaks caused by the Coulomb transitions which are at 12 , 20,38 , and $81 \mathrm{eV}$ for the $\Delta n=1$ transitions $(7-6),(6-5)$, $(5-4)$, and $(4-3)$, respectively.

Cascade calculations have been extended to follow the development of the velocity during the de-excitation cascade and, therefore, predict kinetic energy distributions at the time of X-ray emission from a specific level (extended standard cascade model ESCM [42]). Calculations exist at present only for muonic and pionic hydrogen. Figure 2 shows such a prediction for $\pi \mathrm{H}$ where energies have been scaled to the $\pi \mathrm{D}$ case. It turned out in the analysis of the $\mu \mathrm{H}(3 p-1 s)$ line shape that the first ESCM predictions could not describe the measured spectrum [47]. Therefore, a model independent approach was used to extract the relative strength of Doppler contributions directly from the measured line shape; a method applied successfully first in the neutron time-of-flight analysis [46]. Here, the kinetic

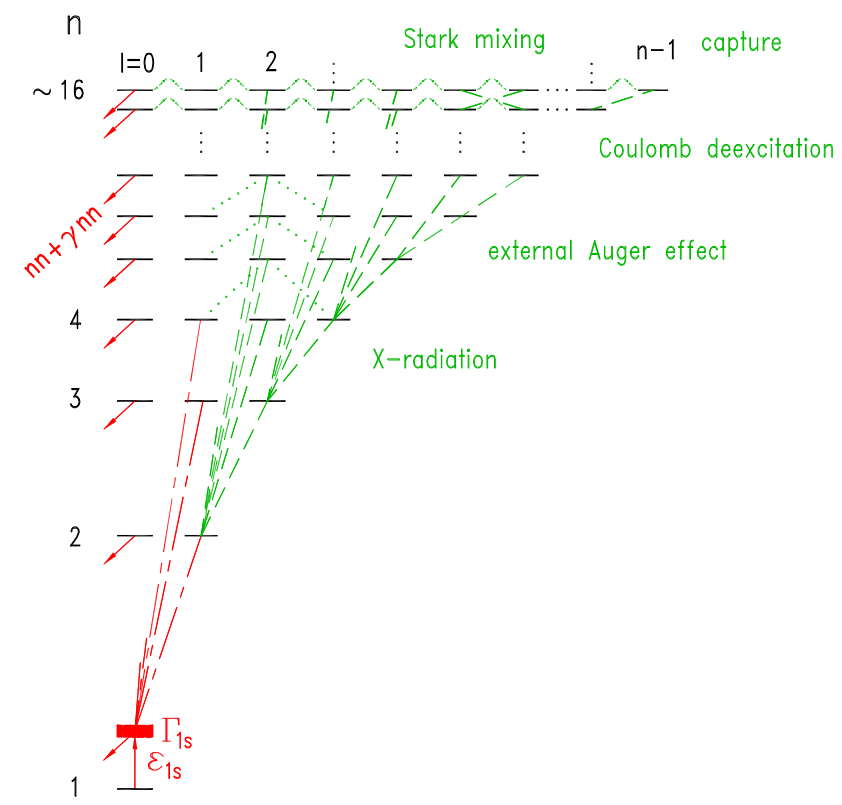

Fig. 1. De-excitation cascade in pionic deuterium. The hadronic shift is defined to $\epsilon_{1 s} \equiv E_{e x p}-E_{Q E D}$, i. e., a negative sign as is the case in $\pi \mathrm{D}$ corresponds to a repulsive interaction.

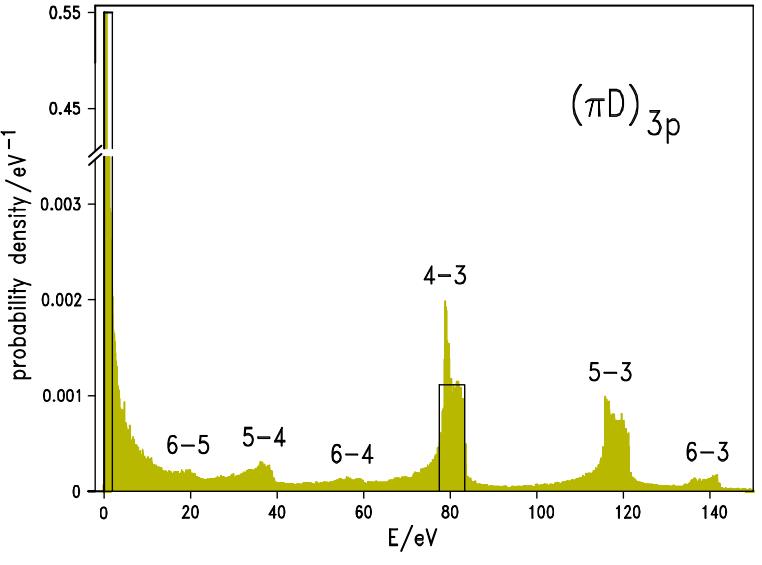

Fig. 2. Prediction for the kinetic energy distribution of the $\pi \mathrm{H}$ atom at the instant of $(3 p-1 s)$ transition scaled to the kinematics of the $\pi \mathrm{D}$ system for a density of 10 bar equivalent. For the analysis, the distribution was tentatively approximated by a box describing the low-energy component and a second one for the high-energy component around $80 \mathrm{eV}$. The low-energy component reaching 0.55 dominates the distribution (note the broken vertical scale).

energy distribution is modeled by boxes around the peaks generated by Coulomb transitions. One approach as used in the analysis of this experiment is included in Figure 2.

In the case of exotic hydrogen, Auger emission is only possible during the collision with other target atoms (external Auger effect). Auger transitions prefer de-excitation steps as small as possible given that the energy gain exceeds the binding energy of the electron. Consequently, Auger emission contributes mainly in the range $n \approx 6-10$ and $\Delta n=1$ transitions are favored [40,42]. Due to the mass difference, Doppler broadening from recoil is negligibly small compared to the one caused by Coulomb transitions.

It is known from muon-catalysed fusion experiments that during $\mu \mathrm{H}+\mathrm{H}_{2}$ collisions metastable hybrid molecules are formed like $[(p p \mu) p] e e[49,50]$. An analogue process is expected in $\pi \mathrm{D}+\mathrm{D}_{2}$ collisions, Such complex' are assumed to stabilise non radiatively by Auger emission. However, radiative decay out of molecular states has been discussed, and its probability is predicted to increase with nuclear mass [49-52]. Possible X-ray transitions from molecular states would falsify the value for the hadronic shift determined from the measured X-ray energy. As molecular formation is collision induced, the fraction of molecules formed and the corresponding X-ray rate should depend on the target density. Therefore, a possiblity to identify such radiative contributions is a measurement of the $\mathrm{X}$ ray energy at three different densities $(3.3,10$, and 17.5 bar equivalent density in this experiment).

\section{Experiment}

The experiment was performed at the $\pi \mathrm{E} 5$ channel of the proton accelerator at PSI which provides a low-energy pion beam with intensities of up to a few $10^{8} / \mathrm{s}$ (Fig. 3). Pions of $112 \mathrm{MeV} / \mathrm{c}$ were injected into the cyclotron trap 


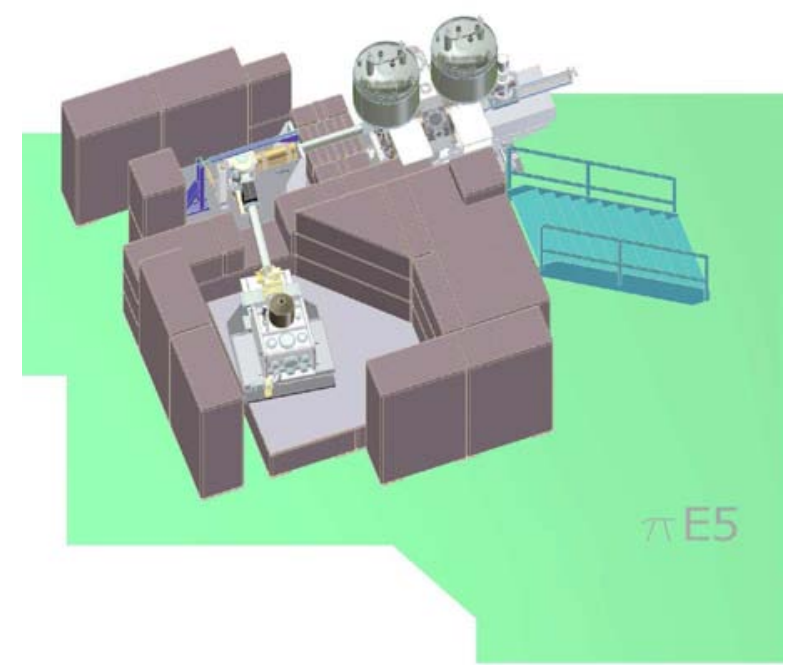

Fig. 3. Setup of the $\pi \mathrm{D}$ experiment in the $\pi \mathrm{E} 5$ area at the Paul Scherrer Insitut (PSI). The roof of the concrete shielding is omitted tp show the vacuum system connecting the cyclotron trap (upper right), crystal chamber (upper left) and the cryostat of the Xray detector (bottom left).

II [1] and decelerated using a set of degraders optimized to the number of pion stops in a cylindrical cryogenic target cell of $22 \mathrm{~cm}$ length and $5 \mathrm{~cm}$ in diameter in the center of the trap. The cell was filled with deuterium gas cooled by means of a cold finger. About $0.5 \%$ of the incoming pions per bar equivalent pressure are stopped in $D_{2}$ gas. Xradiation could exit the target cell axially through a $5 \mu \mathrm{m}$ thick mylar ${ }^{\circledR}$ window. The window foil is supported by horizontal aluminum bars.

The Johann-type Bragg spectrometer was equipped with a spherically bent $\mathrm{Si}$ crystal cut parallel to the (111) plane having a radius of curvature of $R=2982.2 \pm 0.3 \mathrm{~mm}$. The reflecting area of the crystal having $10 \mathrm{~cm}$ in diameter was restricted by a circular aperture to $95 \mathrm{~mm}$ in diameter to avoid edge effects and to $60 \mathrm{~mm}$ horizontally to keep the Johann broadening small $[53,54]$.

Such a spectrometer is able to measure simultaneously an energy interval according to the width of the X-ray source when using a correspondingly extended X-ray detector. Being a pixel device, CCDs are ideally suited for Xrays in the few $\mathrm{keV}$ range because they combine an intrinsic position resolution with the good energy resolution of semiconductor detectors. In addition, the granularity of the CCDs allows for efficient background rejection by means of pattern recognition (cluster analysis). Photo electrons from few-keV X-ray conversion are stopped within a few micrometer and, therefore, deposit charge in one or two pixels with a common boundary. Beam induced background, mainly high energy photons from neutrons produced in nuclear pion absorption and captured by surrounding nuclei, leads to larger structures. Together with the massive concrete shielding (Fig. 3) such events are efficiently suppressed (Fig. 5).

In this experiment, a $3 \times 2$ array of charge-coupled devices (CCDs) was used covering in total $72 \mathrm{~mm}$ in height and $48 \mathrm{~mm}$ in width [55]. Monte Carlo studies show that about $2 / 3$ of the intensity of the reflection is covered by the height of the array. The detector surface was oriented perpendicular to the direction crystal - detector.

The CCDs' depletion depth of about $30 \mu \mathrm{m}$ yields an optimum quantum efficiency in the $3-4 \mathrm{keV}$ range. A pixel size of $40 \mu \mathrm{m}$ provides a two-dimensional position resolution sufficient to measure precisely the shape of the diffraction image. The relative orientation of the individual CCDs as well as the exact pixel size was obtained by means of an optical measurement using a nanometric grid [56]. The CCDs are operated at a temperature of $-100^{\circ} \mathrm{C}$.

$\mathrm{X}$-ray energies of the ground-state transitions in muonic and pionic hydrogen isotopes are in the few $\mathrm{keV}$ range, where no narrow $\gamma$ lines of sufficient intensity are available to be used to determine precisely the function of a lowenergy crystal spectrometer. However, from highly stripped atoms produced in an electron-cyclotron resonance trap (ECRIT) narrow X-ray lines are emitted at high rate.

The spectrometer response was measured using the narrow $M 1 \mathrm{X}$-ray line from helium-like argon of $3.104 \mathrm{keV}$ as described in $[57,58]$ yielding a resolution of $436 \pm 3 \mathrm{meV}$ (FWHM) when scaled to the energy of the $\pi \mathrm{D}(3 p-1 s)$ transition of $3.075 \mathrm{keV}$. This value is close to the intrinsic resolution of $403 \pm 3 \mathrm{meV}$ given by the rocking curve width as calculated for an ideal flat crystal with the code XOP [59].

The response is constructed from the intrinsic properties of the Si (111) material as calculated from the dynamical diffraction theory with the code XOP [59]. The rocking curve is then convoluted with the experiment geometry by means of a Monte-Carlo ray-tracing code (Fig. 4). Deviations from this ideal response are introduced by convoluting with an additional Gaussian contribution which was determined from the measurement of the $M 1$ transition in helium-like argon.

In the Johann set-up the energy calibration must be provided by a reference line of known energy. In order to avoid any mechanical changes, only X-rays having about the same Bragg angles are suitable for precision measure-

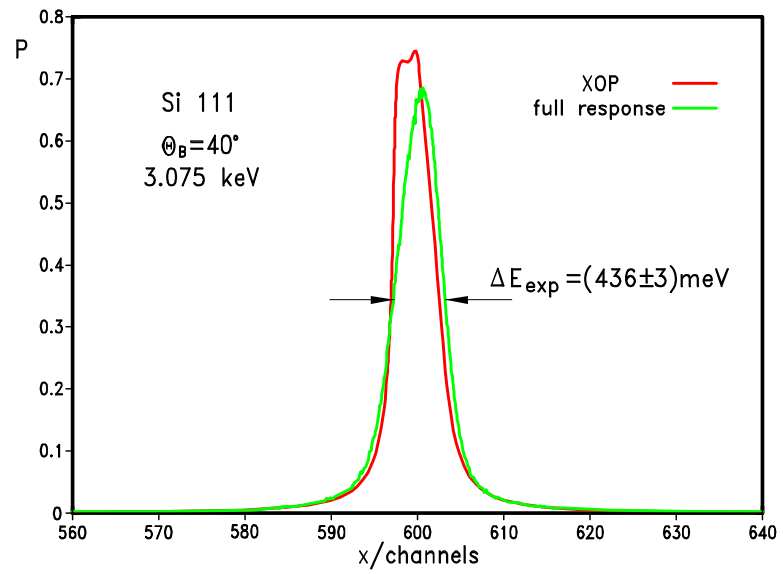

Fig. 4. Transformation of the intrinsic resolution of the Si (111) reflection from a flat crystal by folding with the experiment geometry and an additional Gaussian describing an imperfect mounting. 
$19^{\text {th }}$ International IUPAP Conference on Few-Body Problems in Physics

ments. The best angular matching for the $\pi \mathrm{D}(3 p-1 s)$ measurement was found for the gallium $\mathrm{K} \alpha$ fluorescence lines. For energy calibration, the $\mathrm{Ga} \mathrm{K} \alpha_{2}$ transition (9224.484 \pm $0.027 \mathrm{eV} \mathrm{[60])} \mathrm{was} \mathrm{chosen} \mathrm{because} \mathrm{of} \mathrm{the} \mathrm{smaller} \mathrm{experi-}$ mental uncertainty. The energy of the $\pi \mathrm{D}(3 p-1 s)$ line is obtained by the angular distance to the Ga reference line to be calculated from the position difference of the reflections on the detector and the distance crystal - detector.

The fluorescence target itself was made of a $25 \times 20 \mathrm{~mm}^{2}$ GaAs plate mounted in the rear part of the gas cell but outside the pion stop volume at $82 \mathrm{~mm}$ off the center of the cyclotron trap and away from the crystal. The X-rays were excited by means of an X-ray tube mounted on a window of the cyclotron trap chamber below the gas cell. The value for the tabulated $\mathrm{Ga} \mathrm{K} \alpha$ energies [60] was obtained from measurement also using the compound GaAs [61]. Therefore, a possible chemical shift must not be considered.

The distance center of the crystal to center of the cyclotron trap was $2100 \mathrm{~mm}$ being about $10 \%$ outside the Rowland circle fulfilling the focal condition $R \cdot \sin \Theta_{B}$. The advantage placing the X-ray source off the focal position is that one averages over non uniformities of the target. Both GaAs and $D_{2}$ target are large enough that no cuts in the tails of the reflection occur. The distance crystal - detector, chosen close to the assumed $\pi \mathrm{D}$ focal length, was determined to $\mathrm{y}_{C D}=1918.1 \pm 0.5 \mathrm{~mm}$ by a survey measurement.

Alternating measurements of the Ga fluorescence radiation and the $\pi \mathrm{D}$ line were performed at least once per day (Fig. 7). The Ga fluorescence X-rays together with two inclinometers mounted at the crystal and detector chambers were used to monitor the stability of the line position. Details on the experimental setup maybe found elsewhere [62].

\section{Analysis}

The X-ray detector raw data are made available as the digitalised charge contents and a position index of the pixel. At first, the cluster analysis is performed. As expected, at $3.1 \mathrm{keV}$ only single $(\approx 75 \%)$ or two pixel events $(\approx 25 \%)$ contribute. Hot and defect pixels are masked by software. The cluster analysed charge (ADC) spectra of the CCDs show a pronounced peak originating from $\pi \mathrm{D}$ X-rays on a largely suppressed background (Fig. 5). For each CCD, an individual energy calibration was performed because of the different gain and noise behaviour. The energy resolution in terms of charge is determined by means of a Gaussian fit.

Applying an energy cut in the ADC spectra, an additional background reduction is achieved. Several ADC cuts with a width of $1 \sigma$ to $4 \sigma$ of the respective Gaussian width were used to study the influence of peak-to-background on the result for the hadronic broadening. The minimum relative statistical error is achieved for a cut of $2.5 \sigma$ of the detector's (charge) resolution. With this energy cut, from fits to the position spectra (similar to the one shown in Figure 7 - bottom) count rates of $1448 \pm 49,4010 \pm 74$, and

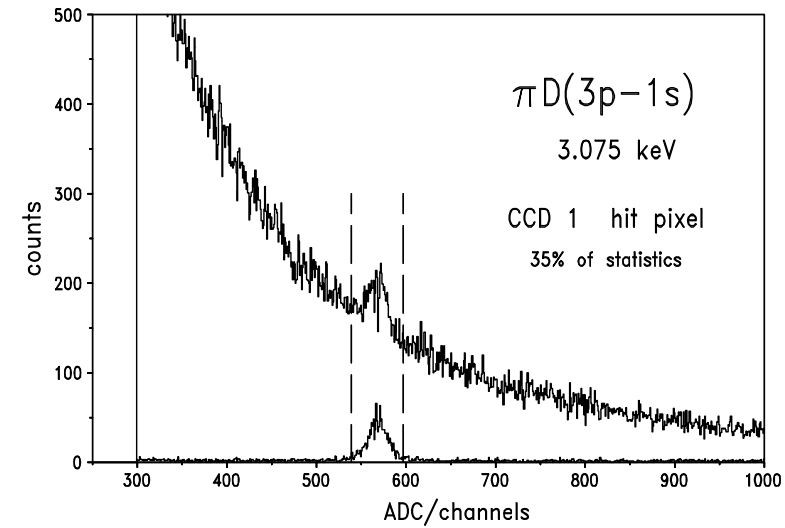

Fig. 5. Background suppression achieved by the cluster analysis of the $\pi \mathrm{D}$ raw data.

$4877 \pm 80$ were obtained for the $\pi \mathrm{D}(3 p-1 s)$ line at the equivalent densities of $3.3,10$, and 17.5 bar, respectively.

In the case of the Ga calibration line, compact clusters up to size 9 were accepted. Because of the larger penetration depth (5 and $105 \mu \mathrm{m}$ for 3.075 and $9.224 \mathrm{keV}$ in silicon [63], respectively), X-rays also convert at the boundary of the depletion region where charge diffusion is already significant. As the calibration measurements were performed without pion beam, the number of background events is negligibly small and, hence, no suppression algorithm is necessary to clean the Ga spectra.

The resulting hit pattern shows curved reflections on the CCD surface. The curvature is corrected for by means of a parabola fit (Fig. 6) before projecting onto the axis of dispersion, which is equivalent to an energy axis.

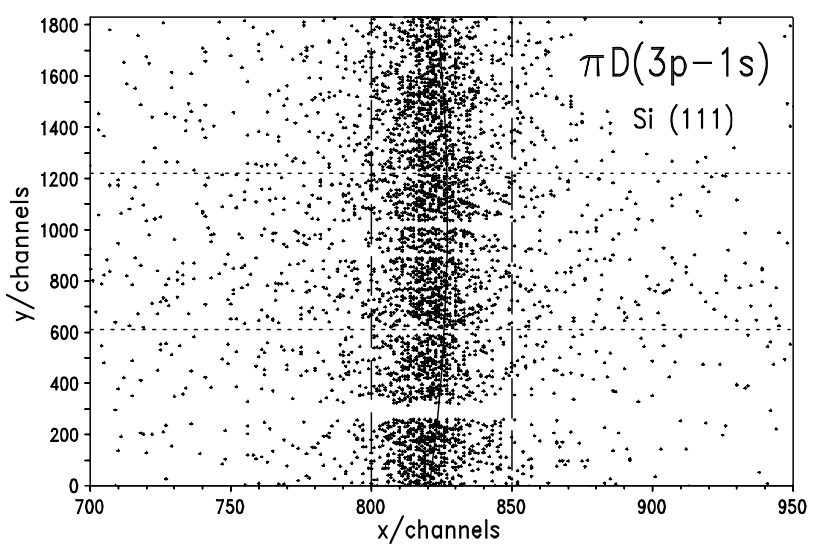

Fig. 6. $\pi \mathrm{D}(3 p-1 s)$ reflection after correction for curvature. The original curvature is indicated by the parabola in the scatterplot of the already corrected data. One channel (pixel) in the direction of dispersion $(x)$ corresponds in first order reflection to $76.402 \pm 0.001 \mathrm{meV}$.

\subsection{Energy calibration}

The position of the $\mathrm{Ga} \mathrm{K} \alpha_{2}$ calibration line was determined applying a single Voigt profile in the fit. The same proce- 
dure was used for the tabulated values of the Ga K $\alpha$ X-ray energies [61].

The $\pi \mathrm{D}$ line was modeled both with a Voigt profile, where Doppler broadening and response function are approximated by a single Gaussian, and secondly, the true response as determined from the ECRIT data convoluted with the imaging properties and including the Doppler contributions from Coulomb de-excitation (Fig. 7). Both methods yield the same position value within a few hundreds of a pixel.

A significant correction to the Bragg angle is the index of refraction shift [59] because the $\pi \mathrm{D}$ and the $\mathrm{Ga} \mathrm{K} \alpha$ line is measured in first and third order, respectively. In addition, bending and penetration correction due to the substantially different energy must be considered. Mechanical shifts from temperature and vibrations are corrected by means of the inclinometer data.

In total, a systematic error of $\pm 8 \mathrm{meV}$ was deduced for the measurement of the $\pi \mathrm{D}(3 p-1 s)$ energy. The combined statistical error obtained as weighted average from the data taken at the three different $D_{2}$ densities amounts
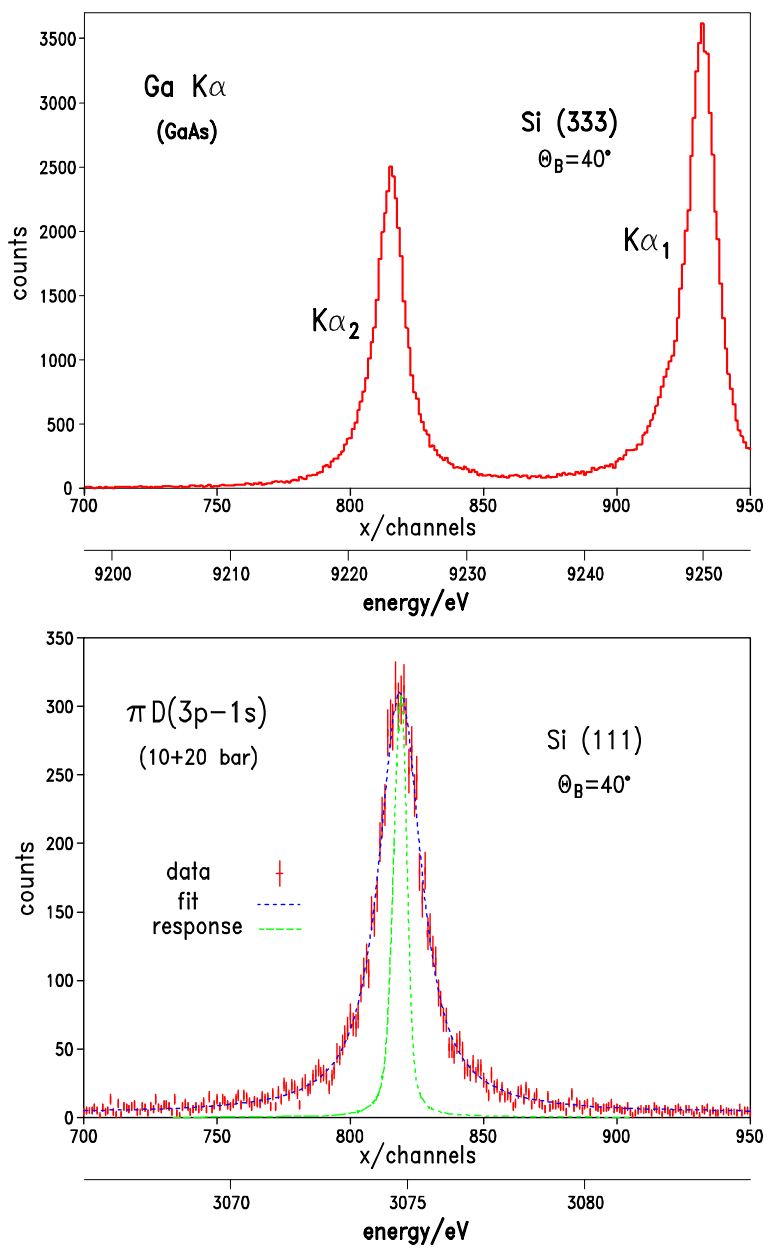

Fig. 7. Ga $\mathrm{K} \alpha_{2}$ calibration line and $\pi \mathrm{D}(3 p-1 s)$ transition measured at an equivalent density of 10 bar. The narrow structure shown inside the $\pi \mathrm{D}$ line represents the resolution of the crystal spectrometer. to $\pm 11 \mathrm{meV}$. The largest contribution to the error stems from the uncertainty of the energy of the $\mathrm{Ga} \mathrm{K} \alpha_{2}$ line of $\pm 27 \mathrm{meV}$. For the transition energy, we find

$$
E_{\pi D(3 p-1 s)}=3075.583 \pm 0.030 \mathrm{eV} .
$$

\subsection{Line width}

To the line shape contribute the response of the spectrometer, the Doppler broadening from Coulomb transitions, and the natural line width of the $\pi \mathrm{D}(3 p-1 s)$ transition. The natural line width is given by the width of the 1s state of about $1 \mathrm{eV}$ because nuclear reactions from the $3 \mathrm{p}$ level are negligible and the radiative width is $28 \mu \mathrm{eV}$.

The model free approach to identify possible Doppler contributions uses narrow boxes of e few $\mathrm{eV}$ width around the kinetic energies as suggested by the energy release of Coulomb transitions. Following the experience from the analysis of the $\mu \mathrm{H}(3 p-1 s)$ line shape, one tries to identify consecutively individual contributions starting with only one contribution of the lowest possible energy, which corresponds to $\pi \mathrm{D}$ systems not accelerated or moderated down by collisions to energies of a few $\mathrm{eV}$ or below. It is sufficient to approximate the various kinetic energy components by box-like distributions as shown in the analysis of the $\mu \mathrm{H}$ experiment.

A $\chi^{2}$ analysis, done by means of the MINUIT package [64], shows that a low-energy contribution is mandatory. It was found that the kinetic energy must not exceed $8 \mathrm{eV}$; a result found independently for taken at 10 bar and the 17.5 bar equivalent density (Fig. 8). The result for the natural line width $\Gamma_{1 s}$ turned out to be insensitive to the upper boundary of the low-energy box for values $\leq 8 \mathrm{eV}$. Therefore, the low-energy component was fixed to a width of $2 \mathrm{eV}$ in the further analysis.

Noteworthy to mention that the ESCM prediction for the kinetic energy distribution for $\pi \mathrm{H}(3 p-1 s)$ case scaled to $\pi \mathrm{D}$ kinematics (Fig. 2) is unable to reproduce the $\pi \mathrm{D}(3 p$ $1 s$ ) line shape because of rather strong contributions of (4$3)$ and $(5-3)$ Coulomb transitions. Searches for any higher energy contributions failed even when using the sum spectrum of the two measurements at 10 and 17.5 bar. This result is rather surprising because also the first analyses of the new pionic hydrogen data show that at least one highenergy component must be assumed to describe the line shape of the $\pi \mathrm{H}(4-1),(3-1)$, and (2-1) transitions [3, 65]. Though a significantly better description of the $\mu \mathrm{H}(3 p-1 s)$ line shape could be achieved after a recalculation of collision cross sections [47,66-68], there is no explanation for the absense of any high-energy components in $\pi \mathrm{D}$.

It has been studied in detail by Monte Carlo which magnitude of high-energy energy components maybe missed with the statistics achieved in this xperiment. The probability to miss a high-energy component around $80 \mathrm{eV}$ (box 2) corresponding to the $(4-3)$ Coulomb transition is displayed in Figure 9. For each set of conditions 400 simulations were performed. It can be seen, that a high-energy contribution of $25 \%$ or larger can hardly be missed. For $10 \%$ relative intensity, the chance is about $15 \%$ which corresponds to about $1 \sigma$ with respect to the full probability 


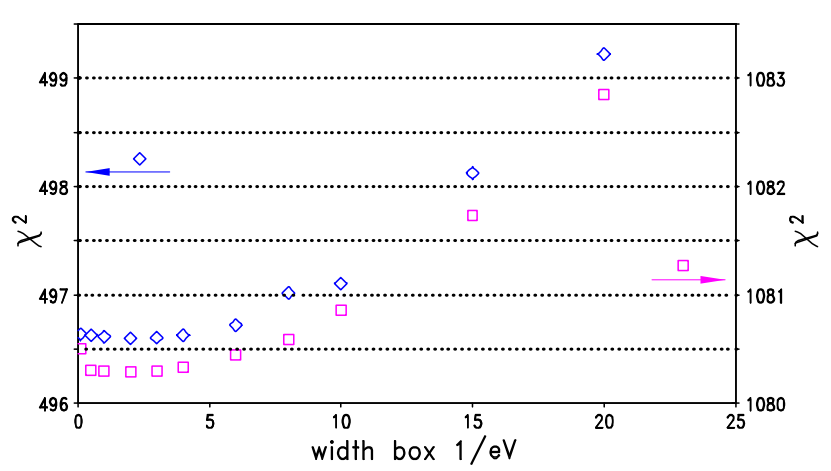

Fig. 8. Search for evidence and width of the low-energy component in the kinetic energy distribution. Diamonds (left $\chi^{2}$ scale) are due to the 10 bar data. Squares (right scale) are from a simultanuous fit to the two spectra taken at 10 and 17.5 bar.

distribution allowing a certain variety for the shape of the box' extension (Fig. 9).

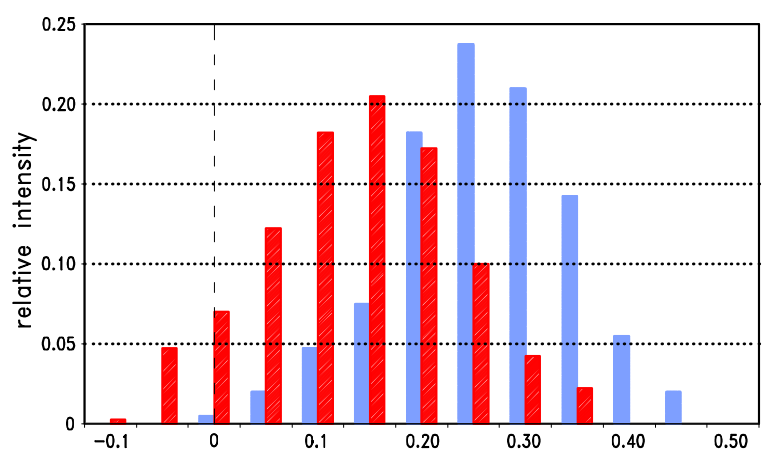

box 2 intensity

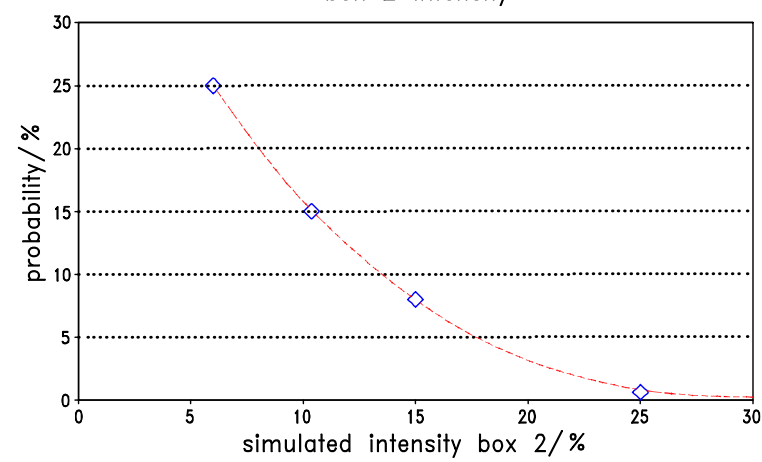

Fig. 9. Top: distributions of relative intensity found from a fit with two boxes when a Doppler contribution of $10 \%$ and $25 \%$ relative intensity, respectively, is assumed. The result is based on 400 Monte-Carlo simulations each performed for the statistics of the sum of the 10 and 17.5 bar data. Bottom: probability to miss a Doppler contribution at $80 \mathrm{eV}$ as a function of its relative intensity.

Assuming weight zero for box 2 results in an upper limit for $\Gamma_{1 s}$, and using the limit of sensitivity of $10 \%$ for a second box yields a lower bound for $\Gamma_{1 s}\left(-\Delta \Gamma_{s y s}\right)$ according to the above-mentioned $1 \sigma$ criterion (Fig. 9). The distribution of the results for the weight a second box (box
2 intensity) and the Lorentz width $\Gamma$ reflects the fluctuations due to the limited statistics (Fig. 10).

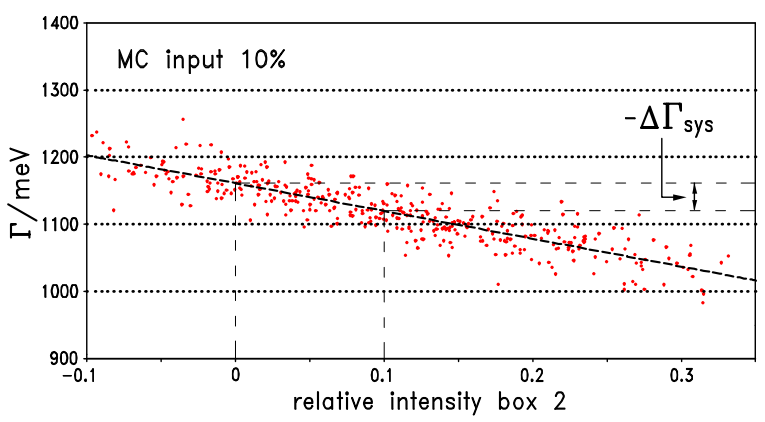

Fig. 10. Distributions of relative intensity of a Doppler box around $T_{\text {kin }}=80 \mathrm{eV}$ of $10 \%$ relative intensity versus the Lorentz width $\Gamma$ extracted from a set of 400 Monte-Carlo simulations performed for the statistics of the sum of the 10 and 17.5 bar data. The input value for $\Gamma=1130 \mathrm{eV}$.

\section{Results}

For extracting the hadronic shift, the pure electromagnetic transition energy has been recalculated to be $\mathrm{E}_{Q E D}$ $=3077.9062 \pm 0.0079 \mathrm{eV}$. The error of this calculation is dominated by the uncertainty of the charged pion mass $(0.0077 \mathrm{eV})[69]$ where the accuracy of deuteron $(0.0012 \mathrm{eV})$ and pion radius $(0.0010 \mathrm{eV})$ contribute only marginal. The numerical accuracy of this calculation is assumed to be better than $1 \mathrm{meV}$ [70].

Combining measured and calculated QED transition energy, we obtain for the hadronic shift

$$
\epsilon_{1 s}=-(2323 \pm 31) \mathrm{meV} .
$$

This result is compared to previous measurements in Figure 11. Note that the new result for the electromagnetic transition energy differs slightly from the values as used in the previous experiments [18,19].

The hadronic broadening is mainly extracted from the sum spectrum of the 10 bar and 17.5 bar measurement using only a low-energy component for the kinetic energy distribution because no density dependence is identified within the experimental accuracy. The combined result

$$
\Gamma_{1 s}=(1171+23) \mathrm{meV} .
$$

is obtained by averaging according to the statistical weight. It is in good agreement with the earlier measurements, but is a factor of about 3 more precise (Fig. 12).

Evaluating the Deser formula, one obtains for the complex scattering length

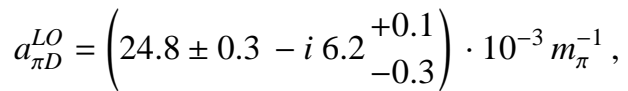




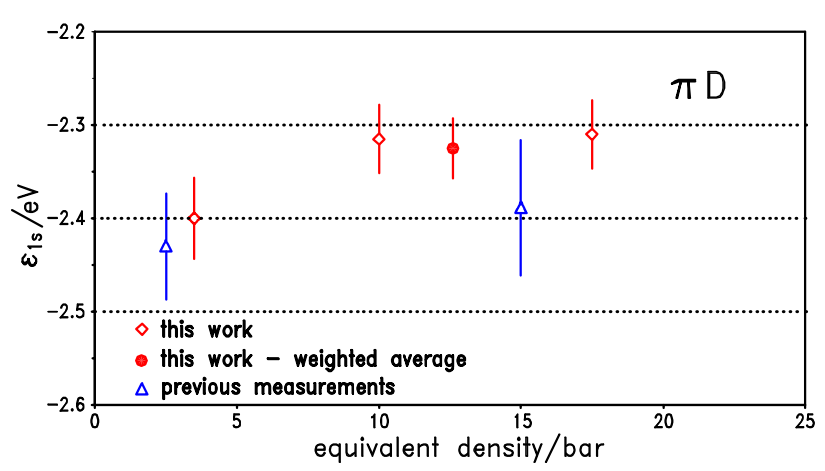

Fig. 11. Experimental information on the hadronic shift in pionic deuterium. The error of the individual measurements at 3.3, 10, and $17.5 \mathrm{bar}$ and, therefore, also of the weighted average is dominated by the uncertainty of the $\mathrm{Ga} \mathrm{K} \alpha_{2}$ energy. The results from the two previous experiments $[18,19]$ are adjusted according to the new values for the pure electromagnetic transition energies.

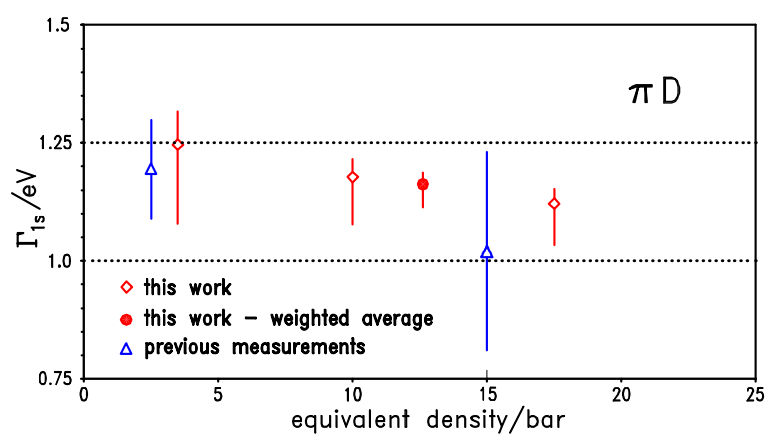

Fig. 12. Experimental information on the hadronic broadening in pionic deuterium. Data points of previous measurements are taken from [18] and [19].

and the value for the threshold parameter $\alpha$ derived from the $\mathfrak{J} a_{\pi D}$ reads

$$
\alpha=\left(\begin{array}{r}
252 \\
-11
\end{array}\right) \mu \mathrm{b} .
$$

The parameter $\alpha$ as determined from pion production experiments shows wide fluctuations even when comparing recent data [71-80]. In some cases, only the statistical error is given for the cross section of the production experiments, but the fluctuations suggest significant systematic uncertainties, which may arise in uncertainties of the normalisation and/or the Coulomb corrections (Fig. 13).

\section{Summary}

The $(3 p-1 s) \mathrm{X}$-ray transition in pionic deuterium has been studied to determine the strong-interaction effects with increased precision. An accuracy of $1.3 \%$ was obtained for the shift, which matches with the theoretical uncertainty of $1.5 \%$ achieved recently $[84,85]$. The experimental uncertainty is dominated by the error of the calibration transition $\mathrm{Ga} \mathrm{K} \alpha_{2}(1.2 \%)$, which could be decreased by a factor

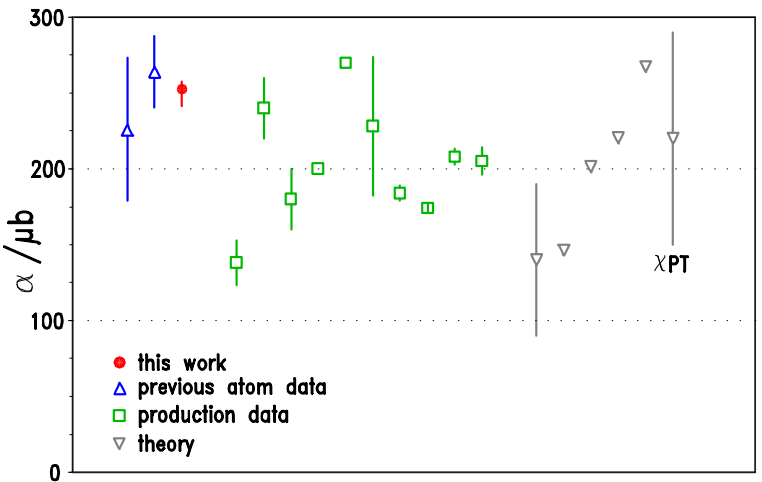

Fig. 13. Threshold parameter $\alpha$ for pion production in the reaction $p p \rightarrow d \pi^{+}$. Input in the Figure (from left to right) are from previous $\pi$ D experiments $([18,19])$, this experiment, pion production and absorption cross sections ([71-74,74-77,79,80]), and a selection of theoretical approaches $([36,81,37,82,83])$.

of about 2 by a remeasurement using a double flat crystal spectrometer in case a significant theoretical progress requires further improvement. The new value for the shift is significantly smaller $(\approx 4 \%)$ than the results reported from the two earlier precision measurements. About $40 \%$ of the discrepancy is due to the new calculation of the $\pi \mathrm{D}(3 p-1 s)$ electromagnetic transition energy.

The theoretical understanding of $N N \leftrightarrow N N \pi$ reaction is continuously increasing. Within the approach of chiral perturbation theory a study of pion production including the next-to-leading order (NLO) terms yields $\alpha^{N L O}=$ $220 \mu b[83,86]$ in good agreement with the pionic-deuterium result (Fig. 13). At NLO the theoretical uncertainty is estimated to about $30 \%$. It is expected to decrease below $10 \%$ within a few years from forthcoming NNLO calculations [85]. Hence, the experimental accuracy of $4.2 \%$ for the hadronic broadening in $\pi \mathrm{D}$ reaches already the expected final uncertainty of the theoretical calculations.

Noteworthy, that at the $10 \%$ level no components from high-energy Coulomb transitions could be identified from the fit to the line shape.

\section{References}

1. PSI experiments R-98-01 and R-06-03, www.fzjuelich.de/ikp/exotic-atoms

2. D. Gotta, Prog. Part. Nucl. Phys. 52 (2004) 133

3. D. Gotta et al., Lect. Notes Phys. 745 (2008) 165

4. S. Deser, L. Goldberger, K. Kaufmann, and W. Thirring, Phys. Rev. 96 (1954) 774

5. T. L. Trueman, Nucl. Phys. 26 (1961) 57

6. G. Rasche and W. S. Woolcock, Nucl. Phys. A 405 (1982) 381

7. D. Sigg et al., Nucl. Phys. A 609 (1996) 310

8. V. E. Lyubovitskij and A. Rusetsky, Phys. Lett. B 494 (2000) 9

9. J. Gasser et al., Eur. Phys. J. C 26 (2002) 13

10. P. Zemp, in Proc. of Chiral Dynamics 2003 , p. 128, Bonn, Germany, September 8-13, 2003, arXiv:hep$\mathrm{ph} / 0311212 \mathrm{v} 1$. 
$19^{\text {th }}$ International IUPAP Conference on Few-Body Problems in Physics

11. G. C. Oades, G. Rasche, W.S. Woolcock, E. Matsinos, A. Gashi, Nucl. Phys. A 794 (2007) 73

12. J. Gasser, V.E. Lyubovitskij, and A. Rusetsky, Phys. Rep. 456 (2008) 167

13. A. W. Thomas and R. H.Landau, Phys. Rep. B 58 (1980) 121

14. T. E. O. Ericson and W. Weise, Pions and Nuclei (Clarendon, Oxford 1988)

15. S. Weinberg, Phys. Rev. Lett. 17 (1966) 616

16. Y. Tomozawa, Nuovo Cim. A 46 (1966) 707

17. D. Sigg et al., Phys. Rev. Lett. 75 (1995) 3245; Nucl. Phys. A 609 (1996) 269; erratum A 617 (1997) 526

18. D. Chatellard et al., Phys. Rev. Lett. 74 (1995) 4157; Nucl. Phys. A 625 (1997) 855

19. P. Hauser et al., Phys. Rev. C 58 (1998) R1869

20. H.-Ch. Schröder et al., Eur. Phys. J C 21 (2001) 473

21. V. V. Baru and A. E. Kudryavtsev, Phys. of At. Nucl. 60 (1997) 1475

22. S. R. Beane, V. Bernard, T.-S. Lee, and U.G. Meißner, Phys. Rev. C 57, (1998) 424

23. A. Deloff, Phys. Rev. C 64 (2001) 065205

24. T. E. O. Ericson, B. Loiseau and A. W. Thomas, Phys. Rev. C 66 (2002) 014005

25. A. Deloff, Fundamentals in Hadronic Atom Theory, World Scientific, London (2003)

26. S. R. Beane, V. Bernard, E. Epelbaum, U.-G. Meißner, and D. R. Phillips, Nucl. Phys. A 720 (2003) 399

27. B. Burasoy and H. W. Grieshammer, Int. J. Mod. Phys. E 12 (2002) 65

28. M. Döring, E. Oset, and M. J. Vicente Vacas, Phys. Rev. C 70 (2004) 045203

29. U.- G. Meißner, U. Raha, and, A. Rusetsky, Eur. Phys. J. C 41 (2005) 213

30. U.- G. Meißner, U. Raha, and, A. Rusetsky, Phys. Lett. B 639 (2006) 478

31. M.P. Valderrama and E. R. Arriola, arxiv:nuclth/0605078 (2006)

32. K. Brueckner, R. Serber, and K. Watson, Phys. Rev. 81 (1951) 575

33. V. L. Highland et al., Nucl. Phys. A 365 (1981) 333

34. D. W. Joseph, Phys. Rev. 119 (1960) 805

35. R. MacDonald et al., Phys. Rev. Lett. 38 (1977) 746

36. A. H. Rosenfeld, Phys. Rev. 96 (1954) 139

37. A. Reitan, Nucl. Phys. B 11 (1969) 170

38. H. Machner and J. Niskanen, Nucl.Phys. A 776 (2006) 172

39. V. Baru, A. Rusetski, priv. comm.

40. E. Borie and M. Leon, Phys. Rev. A 21 (1980) 1460

41. F.J.Hartmann, Proc. of Physics of Exotic Atoms on Electromagnetic Cascade and Chemistry, 1989, Erice, Italy, 1989 (Plenum Press, New York 1990) p. 23 and p. 127, and references therein

42. T. S. Jensen and V.E. Markushin, Eur. Phys. J. D 19 (2002) 165; Eur. Phys. J. D 21 (2002) 261; Eur. Phys. J. D 21 (2002) 271

43. H. A. Bethe and E. E. Salpeter, Handbuch der Physik Band XXXV (Springer-Verlag, Berlin 1957)

44. L. Bracci and G. Fiorentini, Nuovo Cim. A 43 (1978) 9

45. J. B. Czirr et al., Phys. Rev. 130 (1963) 341
46. A. Badertscher et al., Europhys. Lett. 54 (2001) 313, and references therein

47. D. S. Covita et al., Phys. Rev. Lett. 102 (2009) 023401

48. D. S. Covita, PhD thesis, University of Coimbra (Coimbra 2008)

49. D. Taqqu, AIP Conf. Proc. 181 (1989) 217

50. S. Jonsell, J. Wallenius, and P. Froelich, Phys. Rev. A 59 (1999) 3440

51. E. Lindroth, J. Wallenius, and S. Jonsell, Phys. Rev. A 68 (2003) 032502; Phys. Rev. A 69 (2004) 059903(E)

52. S. Kilic, J.-P. Karr, and L. Hilico, Phys. Rev. A 70 (2004) 042506

53. J. Eggs and K. Ulmer, Z. angew. Phys., 20(2) (1965) 118

54. G. Zschornack, Nucl. Instr. Meth. 200 (1982) 481

55. N. Nelms et al., Nucl. Instr. Meth. A 484 (2002) 419

56. P. Indelicato et al., Rev. Sci. Instrum. 77 (2006) 043107

57. D. F. Anagnostopulos et al., Nucl. Instr. Meth. A 545 (2005) 217

58. M. Trassinelli et al., J. Phys., Conf. Ser. 58 (2007) 129

59. M. Sanchez del Rio and R. J. Dejus, Proc. SPIE 3448 (1998) 246

60. R. Deslattes, E.G. Kessler, Jr., P. Indelicato, L. de Billy, E. Lindroth, and J. Anton, Rev. Mod. Phys., vol. 75, no. 1,35 (2003)

61. T. Mooney, priv. comm.

62. Th. Strauch, PhD thesis, Universität zu Köln (Cologne 2009)

63. WM.J. Veigele, At. Data and Nucl. Data Tables 5 (1973) 51

64. F. James and M. Roos, Comput. Phys. Commun. 10 (1975) 343

65. A. Hirtl, PhD thesis, Technische Universität Wien (Vienna 2008)

66. V. N. Pomerantsev and V. P. Popov, JETP. Lett. 83, 331 (2006); Phys. Rev. A 73, 040501(R) (2006)

67. T.S. Jensen, V. N. Pomerantsev, and V.P. Popov, arXiv:0712.3010v1 [nucl-th] (2007)

68. V.P. Popov and V. N. Pomerantsev, arXiv:0712.3111v1 [nucl-th] (2007)

69. C. Amsler et al. (PDG 2008), Phys. Lett. B 667 (2008) 1

70. P. Indelicato, priv. comm.

71. F. S. Crawford and M. L. Stevenson, Phys. Rev. 97 (1955) 1305

72. C. M. Rose, Phys. Rev. 154 (1967) 1305

73. C. Richard-Serre et al., Nucl. Phys. B 20 (1970) 413

74. J. Hüfner, Phys. Rep. 21 (1975) 1

75. D. Aebischer et al., Nucl. Phys. B 108 (1976) 214

76. D. A. Hutcheon et al., Phys. Rev. Lett. 64 (1990) 176

77. B. G. Ritchie et al., Phys. Rev. Lett. 66 (1991) 568

78. D. A. Hutcheon et al., Phys. Rev. A 535 (1991) 618

79. P. Heimberg et al., Phys. Rev. Lett. 77 (1996) 1012

80. M. Drochner et al., Nucl. Phys. A 643 (1998) 55

81. D. S. Koltun and A. Reitan, Phys. Rev. 141 (1969) 1413

82. I. R. Afnan and A. W. Thomas, Phys. Rev. C 10 (1974) 109

83. V. Lensky et al., Eur. Phys. J. A 27 (2006) 37

84. V. Lensky et al., Phys. Lett. B 648 (2007) 46 
EPJ Web of Conferences

85. C. Hanhart et al., in preparation

86. A. Filin et al., Phys. Lett. B 681 (2009) 423 